\title{
USING MODERN INFORMATION TECHNOLOGY TO ENRICH THE PRESENTATION OF RESULTS IN SCIENTIFIC PUBLICATIONS
}

\author{
Gojko Vladić (iD), Dragoljub Novaković (iD), Gordana Delić (iD, \\ Rastko Milošević (iD, Stefan Đurđević (iD) \\ University of Novi Sad, Faculty of Technical Sciences, \\ Department of Graphic Engineering and Design, Novi Sad, Serbia
}

\begin{abstract}
It has been almost two decade since electronic formats entered mainstream in scientific paper publishing. PDF (portable document format) has become without any doubt most used electronic publishing format. Wide adoption of this format offered easy exchange of the information presented conventionally through text and images, which was widely adopted. On the other hand it has been little over a decade since 3D artwork, java script and SWF applications are supported by PDF, but adoption of all of these advanced features is quite low. With technological advancements augmented and virtual reality are becoming useful tools also. Same as in the case of PDF there is the problem, all across the board of scientific fields, of slow adoption of new Information communication technology ICT. This paper aims to explore and present some of the new ICT features that could help improve presentation of scientific results and also to illuminate the reasons they are rarely used in publications.
\end{abstract}

Key words: PDF, augmented reality, multimedia, scientific publishing

\section{INTRODUCTION}

Using text and images in scientific publishing, and publishing in general, has its long history. This way of presenting the content was transferred to internet and electronic publishing. With technological advancements of electronic publishing formats new communication opportunities arouse. While migration to the internet was done quite fast and it was met with enthusiasm, this was not the case with usage of full capabilities of the electronic publishing formats such as Portable Document Format. Furthermore new technologies such as augmented reality are becoming more available and used each day, but their adoption and integration in scientific publications remains insignificant. This paper aims to explore and present features that could help improve presentation of scientific results and also to illuminate the reasons they are rarely used by authors.

\subsection{Portable document format}

Portable Document Format (PDF) can be used to present and exchange electronic content reliably, independent of hardware or software system. Adobe PDF was invented in beginning of ' 90 and by now it became an open standard maintained by the International Organization for Standardization (ISO). It meets ISO 32000 standards for electronic document exchange, including special-purpose standards such as PDF/A for archiving, PDF/E for engineering, and PDF/X for printing and variety of accessibility standards for people with disabilities. PDFs is based on the PostScript language and can contain fonts, vector graphics, raster images, links, buttons, form fields, audio, video, business logic, they can be signed electronically and can be easily viewed using various free software. Java support is present since 2000. and since 2004. PDF files may contain three dimensional objects using U3D or PRC, and various other data formats (Acrobat.adobe, 2018). Despite continuous improvements in the PDF capabilities there is little to no adoption of these capabilities in scientific publishing. Other than information presented conventionally through text and images, advanced features are not used even when there is clear need for them. Good examples are the medical illustrations, which have always been handicapped by being restricted to two dimensions even though three dimensional imaging and scanning is available and used for some time now (Ziegler, 2011; Maunsell, 2010).

\subsection{Augmented and virtual reality}

This interaction technology overlays computer-generated virtual elements onto real-world environment images. Augmented reality became well known technology only with recent advancements in the hardware of the mobile devices. These advancements made it possible to capture the real world images, process them, while simultaneously process the virtual content and to combine them in to augmented 
reality which is shown on the device's display. Concept is based on the information displayed and image overlay which are context-sensitive, depending on the observed objects and by enhancing perception of reality by combining physical and digital content and giving additional value, in information terms. This technology is widely available through different applications, mostly intended for the mobile devices. (Zhu et al, 2004; Azuma, 1997; Azuma et al, 2001). Although there are technological variations, basically augmentation is produced after a series of digital data transformations. First, the real video image is captured and transformed. Then, this image is processed in order to determine position of the markers (containing an image pattern that is compared to patterns stored in a database). Next, the algorithm determines pattern orientation as the base for the coordinates frame and calculates the real position of the digital camera in relation to the physical marker. After that, the virtual objects are placed over the markers, and the final image is rendered and sent to the display (Soares, et al, 2012). Production of the AR content has become fairly easy and there are researchers suggesting that it should become standard practice to be incorporated into figures representing structural data (Wolle et al, 2018).

In contrast to augmented reality, virtual reality completely replaces outside world with computer generated one. Special head set is needed in order to emerge in to virtual world. VR technology is in its first stage of adoption and as such is still rather exotic in hardware and software sense. In the literature overview there are no references of using this technology in order to present scientific data, but there are numerous references for usage in the education.

\section{CONSIDERATION OF THE BENEFITS}

Today scientific research results are or could be captured as three-dimensional (3D) data, for example modern Scanning Electron Microscope SEM equipped with 3DSM solution can produce 3D image in real time and it is possible to obtain a precise quantitative 3D model suitable for visualization and analysis of the 3D surface with generation of complete metrology reports. These 3D scans can be easily presented in the research papers but often they are not.

Good illustration can be found in graphic engendering, as there is often a need for graphical presentation of a colour coordinates or a colour gamut. Both colour and gamut are often presented with still image of Lab colour space, but information value of that image is close to none, as observer can perceive only two dimension and needing all three dimensions in order to comprehend the full information. Using to full extent PDF 3D capabilities can be improve understanding of colour data, as shown in Figure 1. In order to comprehend 3D space at least three orthographic images are needed, but problems with size and legibility are apparent. Interactive $3 d$ representation showing colour space with colour position can be rotated and zoomed in order to get a real sense of colour positions in Lab colour space.

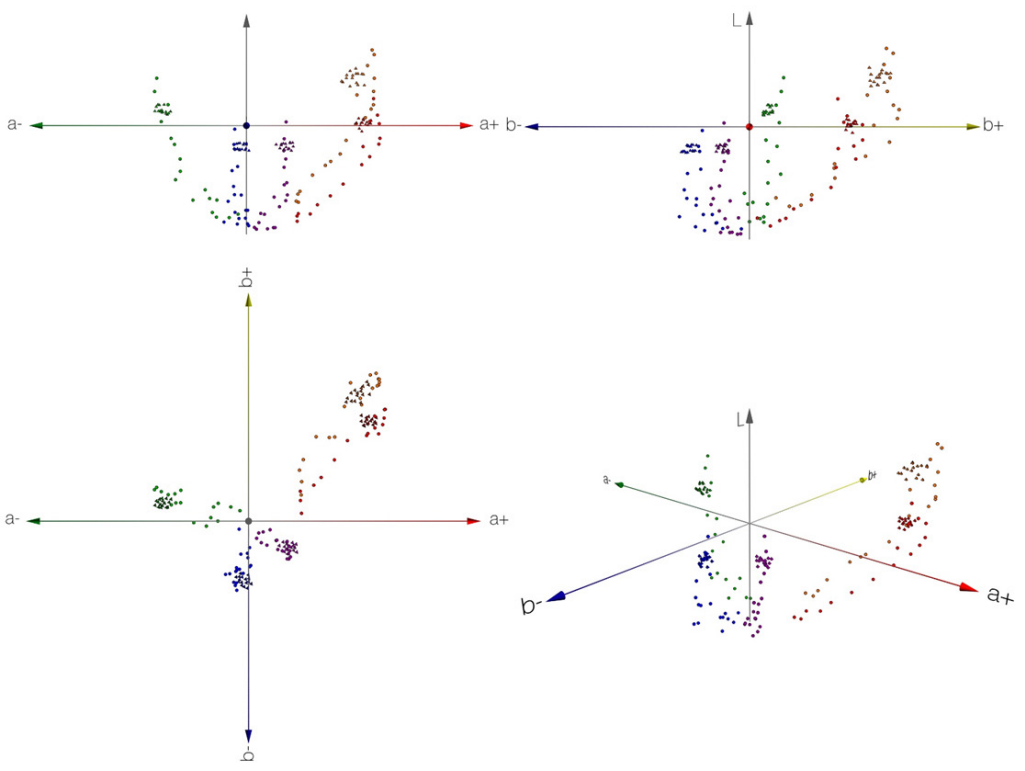

a)

Figure 1 (part 1): a) Static orthographic images of Lab colour space 


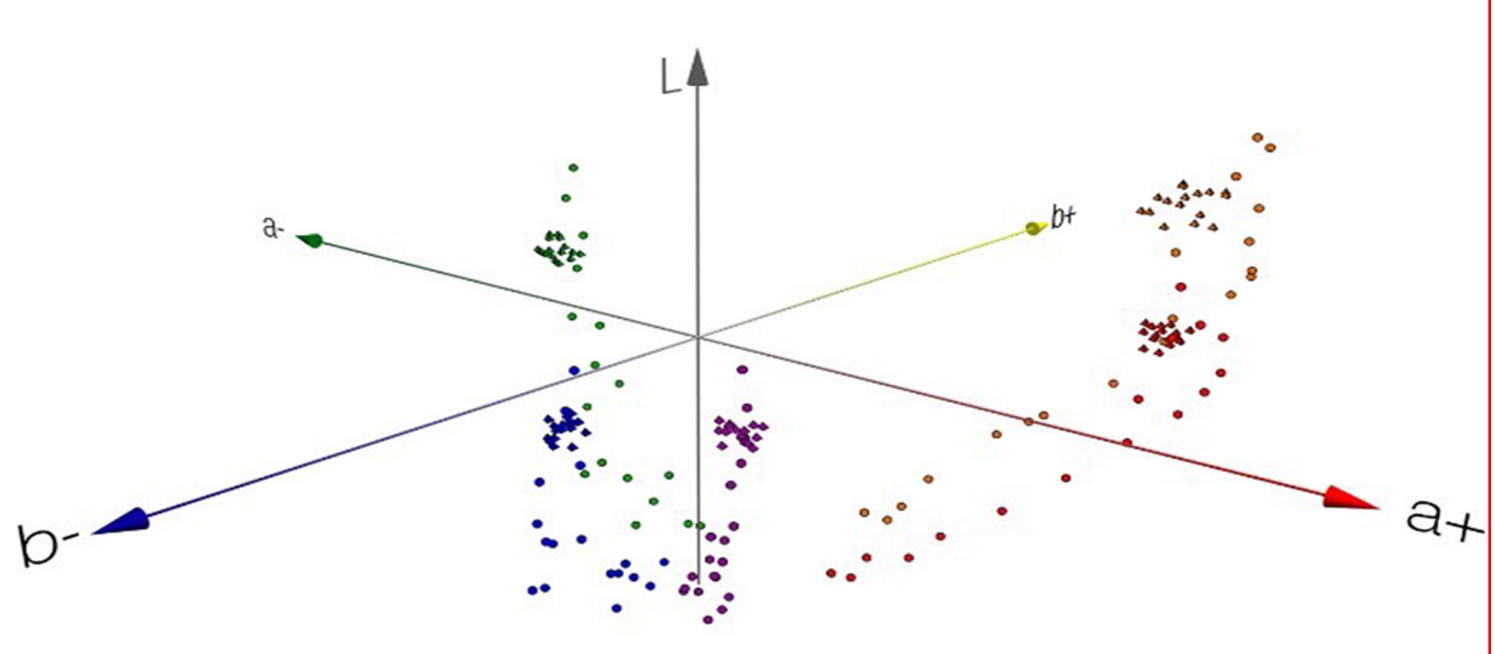

Figure 1 (part 2): b) Interactive 3D representation

Click the image for $3 D$ in the electronic edition of proceedings

This is achieved by constructing 3D model of Lab space, using specialized colour analysis application capable of 3D visualization or any 3D modelling application by imputing colour coordinates.

In case of PDF, universal three-dimensional (U3D) or PRC file format must be used for interpretation by PDF. Universal 3D is a binary file format that contains all necessary information to describe a 3D scene graph. This includes the geometry data, palette definitions, lighting, cameras ("views"), texturing and pre-defined animations ("motions") (Newe, et al, 2013). U3D is a compressed file format standard for 3D computer graphics data, standardized by Ecma International in 2005 as ECMA-363 in order to facilitate data exchange. It can be inserted into PDF documents and interactively visualized by Acrobat Reader, since version 7. PRC is older file format which has been pushed aside by more popular U3D.

PDF format has integrated support for video (including H.264), audio, and other multimedia. Adding the video and other multimedia to the scientific publication can vastly improve presentation of experimental methodology, results, etc. Figure 3 shows the static frame (image of the break in the tensile test of ABS and PLA 3D printed sample, but in electronic version of the proceedings break can be observed in real time and important differences between two materials can be seen, which are omitted in static image.

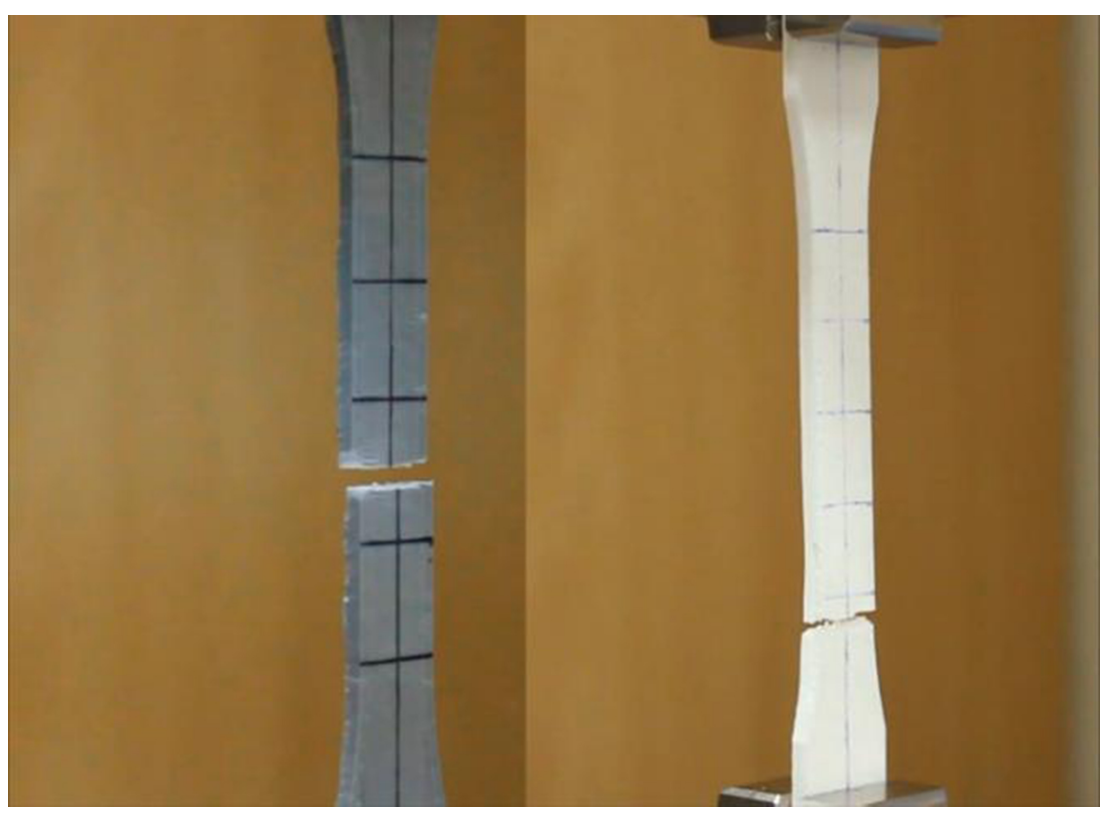

Figure 3: Static images of a tensile test, Click the image for video in the electronic edition of proceedings 
PDF also supports attachment of Adobe Flash applications shockwave file (SWF). This format offers integration of the interactive application suitable for presentation of interactive graphs and great verity of other use. Over the time SWF became obsolete due to security issues and is really supported without separate installation for operating system or internet browsers.

Similar results can be achieved by using augmented reality technology with the difference that content is not embedded in to the PDF file for witch viewer needs to be installed on to the device, but in the separate application. This application is produced for each platform iOS, Android or Windows in which $3 \mathrm{D}$, video, or other content is triggered by the image. This offers possibility for seamless combination of printed media and multimedia content. Such content can be manipulated and navigated using touch screen of the mobile device or other AR device, as shown on augmented 3D graph example of Graphmented app, figure 2.

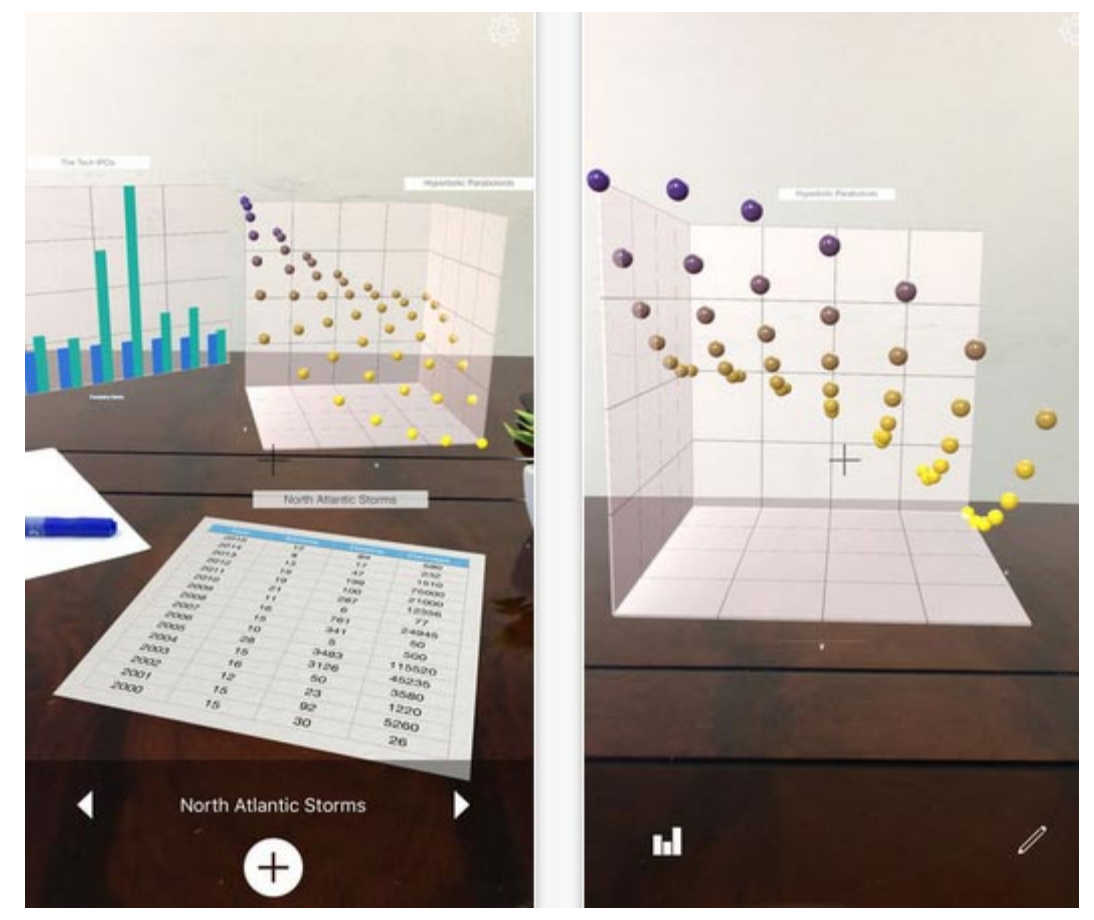

Figure 2: Graphmented app example of augmented reality data presentation

Alongside simple mobile applications such as Graphmented, augmentation can be achieved by using much more versatile software such as Unity and Vuforia combination which enables video and other multimedia formats to be included in augmented reality application, with addition of potential for full customization of the application.

Usual process for generating augmented reality application consists of material preparation. Makers, or triggers must be well defined imaged with contrast areas in order to be recognized by the machine. Alternative is to use dedicated $Q R$ codes which in case off Apple phones offer automatic play of augmented content even without specialized application.

Procedure for generating augmented reality applications starts with preparation of 3D models following standard procedure of geometry generation and optimization for reproduction on a mobile device, although most modern mobile devices have powerful graphic hardware that can render very complex 3D scenes. Depending on the platform certain format of 3D files must be saved. Preparation of video or other media content is done with usual editing software and saved in appropriate format. Content can be stored in the application which enables offline use, or it can be uploaded on the internet and downloaded by the application at time of use. When markers and content is ready they are connected using software such as Unity for creation of the application interface interactivity and 3D environment, and Vuforia engine for easy insertion of augmentation. 


\section{CHALENGES IN ADOPTION AND IMPLEMENTATION}

Spatial presentation, multimedia and possibility to interact with data representation offers better insight and encourages curiosity. Technical possibility for all of this is present in the most popular electronic publishing format, used by more than 500 million users worldwide, for more than a decade. Big publishers promoted and encouraged authors to embed 3D data directly into their publications, Elsevier "Authors of selected Elsevier journals are invited to enrich their online articles by providing supplementary 3D models" yet there is very little content supported with this technology. Augmented reality in comparison to PDF is still novelty and used seldom. Having all this in mind, it must be asked "Why don't authors use the advantages?".

In informal interviews different reasons were stated for not using all the capabilities of available technology. Reasons differed depending on the field of study, majority of authors do not know about the possibilities at all, and when introduced to the possibilities did not recognize potential in their field. Skills required for preparation of content are also deterring factor, as academics in most scientific field are not familiar with 3D modelling, multimedia production or trained for it.

Relative obscurity of U3D file format and lack of support by the mayor 3D modelling applications is also one of the reasons among authors who are familiar with the PDF format possibilities. Specialized conversion applications are often needed as there is no possibility to directly save U3D format. Although, this is somewhat emended by variety of online convertors.

Closed file formats of laboratory equipment and software manufacturers could also be significant factor. For example MRI, CT or ultrasound in medical science often produce DICOM format which is hard to translate in to other formats and sometimes it is hard to even view them using independent software. Last factor are the editors and reviewers of scientific publications who do not promote and insist on presentation of results in the best way possible, by using up to date technologies.

All the previously mentioned obstacles are in relation to PDF format, while augmented reality technology is considered as a game like feature, and majority of scientific authors are unfamiliar with the process of creating augmented reality application and content.

\section{CONCLUSIONS}

Despite obvious advantages of using modern information communication technology to enrich the presentation of results in scientific publications examples are seldom encountered. 3D data is produced as a result of research, but not presented in that form, instead it is reduced to 2D imagery. This results in loss of valuable information. Technology to support better presentation of scientific research is present and it should be used to its full potential. Obstacles discussed in this paper could be overcame by proper education, promotion and stimulation of scientific authors and journal editors. Having in mind tough road of full PDF features adoption, it can be foreseen that augmented reality will not be significantly present in scientific publications for a long time.

\section{ACKNOWLEDGMENTS}

The research is supported by the Ministry of Education, Science and Technology Development of the Republic of Serbia, project number: 35027 "Development of software model for scientific and production improvement in graphic industry".

\section{REFERENCES}

[1] Acrobat, URL: https://acrobat.adobe.com/us/en/ (last request: 2018-10-12).

[2] Azuma R, Baillot Y, Behringer R, Feiner S, Julier S, Maclntyre, B.: "Recent Advances in Augmented Reality", IEEE Computer Graphics and Applications 21(6), 34-47, 2001. doi: 10.1109/38.963459

[3] Azuma, R.: "A Survey of Augmented Reality", Teleoperators and Virtual Environments, 6(4), 355-385, 1997, doi: 10.1162/pres.1997.6.4.355

[4] Maunsell J.: "Announcement regarding supplemental material", The Journal of Neuroscience, 30(32), 10599-10600, 2010, URL: http://www.jneurosci.org/content/30/32/10599.short (last request: 2018-08-15) 
[5] Newe, A., Ganslandt, T.: "Simplified Generation of Biomedical 3D Surface Model Data for Embedding into 3D Portable Document Format (PDF) Files for Publication and Education", PLoS ONE 8(11), e79004, 2013. doi: 10.1371/journal.pone.0079004

[6] Soares, A, Andrade, A., Lamounier, E., Cardoso, A.: "Virtual and Augmented Reality: A New Approach to Aid Users of Myoelectric Prostheses", 2012. doi: 10.5772/50600

[7] Wolle, p., Müller, M. P., Rauh, D.: "Augmented Reality in Scientific Publications-Taking the Visualization of 3D Structures to the Next Level", ACS Chemical Biology, 13(3), 496-499, 2018. doi: 10.1021/acschembio.8b00153

[8] Zhu, W., Owen C. B., Li H., Lee J.: "Personalized In-store E-Commerce with the PromoPad: an Augmented Reality Shopping Assistant", Electronic Journal for E-commerce Tools and Applications, 1(3), 1-19, 2004. doi=10.1.1.83.8198

[9] Ziegler, A., Mietchen, D., Faber, C., Hausen, W., Schöbel C., Sellerer, M., Ziegler, A.: "Effectively incorporating selected multimedia content into medical publications", BMC Medicine, 9, 17, 2011. doi: 10.1186/1741-7015-9-17

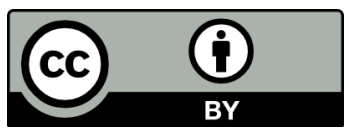

(C) 2018 Authors. Published by the University of Novi Sad, Faculty of Technical Sciences, Department of Graphic Engineering and Design. This article is an open access article distributed under the terms and conditions of the Creative Commons Attribution license 3.0 Serbia (http://creativecommons.org/licenses/by/3.0/rs/). 